\title{
Mhcj

\section{Para reflexionar: Tendencias cinematográficas en América Latina y Gestión de las sociedades postmodernas}

\section{To reflect: Cinematographic tendencies in Latin America and Management of postmodern societies}

Dra. Montserrat Jurado Martín | mjurado@umh.es

Co - editora de Miguel Hernández Communication Journal

Miguel Hernández Communication Journal es una revista internacional de índole científica especializada en la comunicación en sus distintas vertientes como la periodística, audiovisual y persuasiva, contemplándose también otras temáticas interdisciplinares relacionadas con la comunicación. MHCJ pertenece a la Plataforma Latina de Revistas de Comunicación, PLatCom, y su vocación es la de servicio público a las comunidades de investigadores para difundir sus resultados científicos.

En este número 8 de 2017 se reúnen artículos de gran calidad e interés científico que tratan diversos aspectos de la comunicación e, incluso, su transversalidad con otras ciencias. De esta manera, MHCJ aporta su granito de arena a la investigación académica sirviendo de escaparate y reflejo de los temas que interesan y preocupan a los investigadores tanto nacionales como internacionales en este ámbito.

La revista se encuentra en un periodo de trasformación y adaptación siguiendo la estela de otras grandes de su ámbito y tiene por objetivo convertirse en una publicación anual con dos momentos de edición: en enero y julio. En ese intervalo de tiempo y reajuste, este número publicará excepcionalmente su versión digital en el mes de julio y adelantará a septiembre su cierre y de esta manera iniciar el trabajo de la publicación de 2018 a enero. Esta acción supone un gran esfuerzo del grupo de editoras que trabajamos al otro lado y sin duda de nuestros revisores a quiénes aprovechamos para agradecer infinitamente todo su tiempo y dedicación altruista por la causa: la publicación en abierto y 
de calidad de trabajos de investigación de profesionales ya consagrados y de otros que están en el buen camino.

Tal y como anunciamos en la edición de 2016, MHCJ publicará dos monográficos anuales en cada número. En esta ocasión, el primero de los monográficos, lleva por título Panorámica y Tendencias de los Estudios Cinematográficos en América Latina en el Siglo XXI. Está coordinador por la maestra Estela Solís Gutiérrez de la Universidad Autónoma de Baja California (Mexicali, México) y el doctor Lauro Zavala Alvarado de la Universidad Autónoma Metropolitana de Xochimilco (México). Se trata de trabajos caracterizados por la recopilación de bibliografía sobre el tema en cada uno de los países que finalmente participaron en el monográfico: México, anfitrión del monográfico; Argentina, Chile y Brasil.

Se quedaron en el camino de este monográfico otras propuestas que llegaron a la revista $y$, aunque interesantes desde el punto de vista documental y divulgativo, o no reunían los rasgos propios de los artículos de investigación en revistas académicas o fueron rechazados en la revisión ciega por pares. No nos enorgullece airear que el rechazo de la revista ha afectado a los contenidos de un monográfico del que puede pensarse a priori que selecciona, por parte de sus coordinadores, a los autores buscando una combinación entre su especialización y su calidad como investigadores, pero es el precio que se paga por la búsqueda de la calidad en los contenidos y el rigor en el proceso de publicación. Esta circunstancia supuso un retraso inusual de la publicación prevista en enero de MHCJ en un momento de transición y apuesta por la calidad. Pero las prisas no son buenas compañeras cuando se quiere hacer un buen trabajo y hoy podemos estar satisfechos de los resultados.

Así y todo los textos que finalmente integran este monográfico son: Panorama sobre la situación de los estudios de cine en Argentina a partir del año 2000, de Alejandro Kelly Hopfenblatt; Los estudios sobre cine en México al inicio del nuevo siglo, de Lauro Zavala Alvarado; Los estudios cinematográficos en Chile: aproximaciones a la conformación y reflexión de campo, de María Marcela Parada Poblete; y Para além do ano 2000, pensar cinema no Brasil, de Antônio Carlos Amancio y Fabián Núñez.

El segundo de los monográficos recoge una gran variedad de resultados de investigaciones en torno al tema La gestión de la influencia y la reputación en las sociedades postmodernas/líquidas, coordinado por la doctora María José CerdáBertomeu. Este volumen reúne interesantes trabajos que abarcan un gran abanico de contenidos en relación al tema propuesta y que son los que siguen: El proceso de cartelización en entornos institucionalizados: legitimación y comunicación política, de Gonzalo Pardo Beneyto y María Ángeles Abellán López; Ceremonial y protocolo: el tránsito al posmodernismo, de Serafín Piñeiro Rodríguez; Millennials y redes sociales: estrategias para una comunicación de marca efectiva, de José Javier Ruiz Cartagena; Transparencia y riesgo reputacional en las políticas públicas de Estado, de 
Ana Tomás López; Comunicación y Participación en Entornos Virtuales, de Mario Guilló y José F. Mancebo-Aracil; Reputação corporativa na Web: reflexões acerca da formação em Comunicaşão e da articulação de redes sociais no BrasilGrieco, de Fabiana Grieco Cabral de Mello Vetritti y Rafael Vergili.

Presentamos finalmente el bloque de artículos Miscélanea, donde la variedad abarca los ámbitos del audiovisual, el cine, festivales de cine, medio impreso, entre otras. Los textos previstos en esta sección serán publicados en dos momentos, un primer bloque que se presenta en este número de julio y un segundo bloque, en estos momentos en proceso de revisión, que se sumarán en septiembre y con el que cerraremos el número 8 de 2017.

De este primer bloque contaremos con los siguientes trabajos: El uso del cortometraje como estrategia de branded content, de María Dolores Lorán Herrero; Resignación y persistencia en el campo del periodismo: una aproximación a la ideología ocupacional de los periodistas en el estado de Sonora, México, de Alejandra Meza Noriega; Trasladar la partida: Modelos y estrategias de la industria cinematográfica frente a la adaptación de un videojuego, de Mario-Paul Martínez Fabre; El cineminuto: surgimiento, festivales y contenidos de un género audiovisual emergente, de Jacob Bañuelos Capistrán y Carlos Saldaña; y El fracaso del periódico Ahora, el ocaso de la prensa impresa española, de Pedro Vázquez-Miraz.

Esperamos que este número sea del gusto de nuestros lectores y que anime a quiénes no nos conozcan a sumarse a nuestra revista, bien como lectores, autores o revisores. A este último grupo, aunque no podemos cubrir con los honorarios que corresponde las horas que dedican a la revisión de textos, les premiamos a final de año con nuestro Premio al Mejor Evaluador del año valorado en 300 euros. Ahí queda...por si sirve de aliciente.

Gracias. 


\section{(c) (1) () \\ BY SA \\ Licencia Creative Commons \\ Miguel Hernández Communication Journal \\ mhcj.es}

\section{Cómo citar este texto:}

Montserrat Jurado Martín (2017): "Para reflexionar: Tendencias cinematográficas en América Latina y Gestión de las sociedades postmodernas", en Miguel Hernández Communication Journal, nº, pp. 7 a 10. Universidad Miguel Hernández, UMH (Elche-Alicante). Recuperado el __ de de 20 de: link del artículo en mhjournal.org]

\section{How to cite this text:}

Montserrat Jurado Martín (2017): “To reflect: Cinematographic tendencies in Latin America and Management of postmodern societies" en Miguel Hernández Communication Journal, nº, pp. 7 to 10. Universidad Miguel Hernández, UMH (Elche-Alicante). Accessed 20_ in: [paper link in mhjournal.org] 